\title{
Design of Missile with Two-Loop and Autopilot Yaw Using Root Locus
}

\author{
Hamimi Osama Abdelaziz ${ }^{1}$, Muawia M. Ahmed ${ }^{2}$, Dalia Mahmoud ${ }^{3}$ \\ ${ }^{1}$ P.G. Student, Department of Control Engineering, Faculty of Engineering College, Al Neelain University, Khartoum, Sudan \\ ${ }^{2}$ Associate Professor, Department of control Engineering, Faculty of Engineering, Al Neelain University, Khartoum, Sudan \\ ${ }^{3}$ Assistan professor Department of control Engineering, Faculty of Engineering, Al Neelain University, Khartoum, Sudan
}

\begin{abstract}
The objective of this paper is to design an Automatic Landing Systemfor MISSILE based on Root Locus control system. The control method is used to define gains of the controllers to apply the Root Locus method. The block diagram of the proposed control system with required controller gains is presented. Based on automatic control principlesthe transfer functions for open and closed loop are obtained. The Root Locus for open loop is drawn and then gain (K) values are found for given damping ratios. Finally, the step responses of the closed-loop system with automatic landing system controller auto-pilot were drawn. The simulation results have proven the effectiveness of the proposed control system in terms of fast response in the presence of external disturbances..
\end{abstract}

Keywords: MISSILE, Two-loop autopilot, Yaw Attitude, Root-Locus

\section{Introduction}

Autopilot is an automatic control mechanism for keeping the spacecraft in a certain desired flight path and level. In a missile, an autopilot is a closed loop system and it is a minor loop inside the main guidance loop. When the missile carries accelerometer and rate gyros to provide an additional feedback to the missile servos to adjust the motion of the missile, then the control system of the missile is called an autopilot. When the autopilot controls the motion in the pitch and the yaw planes, they are called lateral autopilot. Autopilots are identical in a symmetrical cruciform missile pitch and the yaw. The guidance system detects the height of the missile and that whether the missile is too high or too low, or if the missile is moving too much to the left or right. It measures the deviation or errors and sends these signals to the control system to minimize the errors[1]. Classical control techniques dominate the designs ofMissile and aircraft autopilot. They require a higher tuning and modification sad hoc cannot be avoidable [2]. In the majority of the previous published work on autopilot design, they have considered modeling, design and analysis of autopilots for atmospheric flight vehicles such as guided missiles. As a result, most of the autopilot analysis and design techniques, which are considered in the literature, use classical control approach, such as single input/single output SISO transfer functions characterization of the system dynamics and Bode, [root-locus]. These techniques are valid for a limited set of flight regimes and their extension to cover a wider set of flight regimes and airframe configurations require autopilot gain and compensation network switching [3]. As with phase lag-lead compensation, generally the purpose of lag-lead compensator design in the frequency domain is to satisfy the specifications on steady-state accuracy and phase margin. Alsothere is a specification on gain crossover frequency or closed-loop bandwidth in terms of implicitly or explicitly. The requirement on relative stabilitycan be represented by phase margin specification due to the pure time delay in the system, and they can represent the desired transient response characteristics converted from the time domain into the frequency domain. The speed of response requirements is represented by a specification on bandwidth or crossover frequency in the time domain or a frequency domain requirement on which sinusoidal frequencies will be passed by the system without significant attenuation [4].

\section{Missile Model}

This section discusses a model of a nonlinear missile for an acceleration controller design. In skid-to-turn (STT) cruciform-type missile systems, the missile motion can be classified into two perpendicular channels: the pitch and the yaw motions. A nonlinear missile model in the pitch motion is shown in Fig. 1.

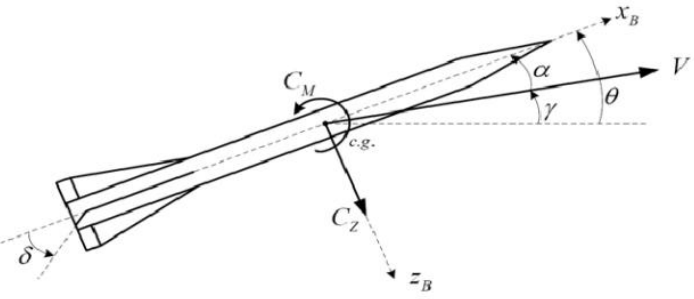

Figure 1: The missile axes and the definition of dynamic variables.

Where $\alpha$ and $\mathrm{q}$ are the angle-of-attack and the body pitch rate, which are the state variables. The control fin, denoted by $\delta$, is the control input. The acceleration component of $Z$ (i.e., aZ) is to be controlled. The notations of $\mathrm{M}, \mathrm{Q}$, and $\mathrm{V}$ represent Mach number, dynamic pressure, and velocity, respectively. The other parameters are the specifications of the missile: reference area $\mathrm{S}$, reference length 1 , mass $\mathrm{m}$, and pitching moment of inertia yy $\mathrm{I}$. The aerodynamic coefficients described by $\mathrm{CZ} 0, \mathrm{CZ} \delta, \mathrm{CM} 0, \mathrm{CM}$ q, and $\mathrm{CM} \delta$ are dependent on Mach number and angle-of-attack, and these parameters can be mostly regarded as continuous functions of their arguments. The aerodynamic coefficients are measured from wind tunnel tests, and these values may contain some errors as compared with true values because of 


\section{International Journal of Science and Research (IJSR) \\ ISSN (Online): 2319-7064}

Index Copernicus Value (2013): 6.14 | Impact Factor (2015): 6.391

the imperfection of the measurements. These errors can be modeled as multiplicative uncertainties:

$$
C_{(0)}^{\text {pert }}=\left(1+\Delta_{\text {pert }}\right) C_{(0)}
$$

Where $\mathrm{C}(0) \mathrm{i}$ are the true aerodynamic coefficients, and $\mathrm{C}(0)$ Pert are the measured aerodynamic coefficients. $\Delta$ pert represents the admissible uncertainties, and their maximum values are about $0.2 \sim 0.3$. The coefficients $\Delta \mathrm{CZ}$ and $\Delta \mathrm{CM}$ represent the aerodynamic coefficients due to the crosscoupling effect of the missile motion, which are considered as additive uncertainties and are mostly continuous functions of Mach number and angle-of-attack[5]. The following block diagrams (fig. 1 represents the transfer function model of flight path rate demand two- loop auto-pilot in yaw plane) [6].

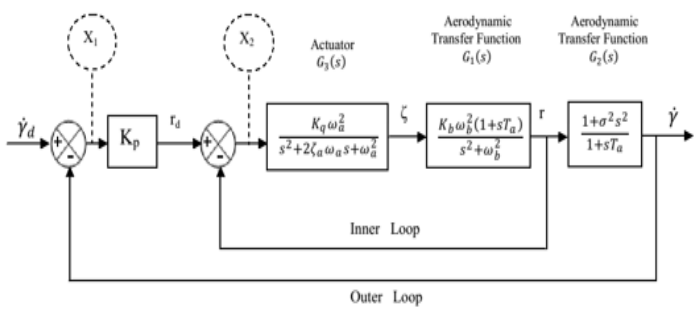

Figure 2: Flight path rate demand autopilot in Yaw plane.

\section{Methodology}

The methodology presented in this paper is firstly the modeling of the system which includes: setting of the missile equations of the motion and derivation of the transfer function, then comes the setting of the requirement and testing the controller with different gains and analyzing the results to obtain the final desired controller.

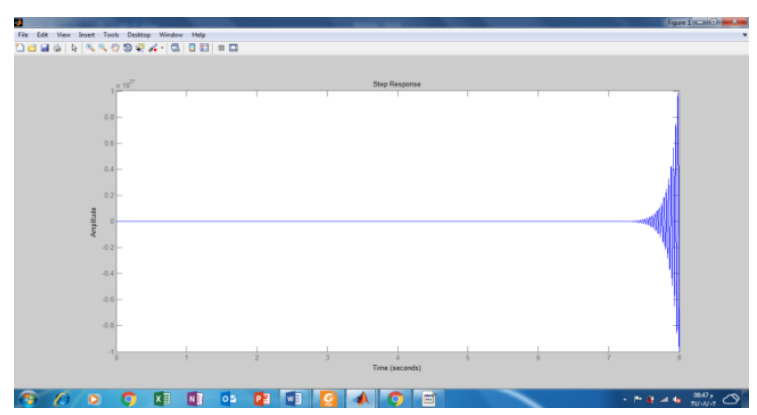

Figure 3: Step response of original Two loop autopilot

\section{Design requirements}

The objective is to design a longitudinal autopilot for a tail governed missile. More specifically, use the tail deflection to track an acceleration maneuver with a time constant of less than $0.35 \mathrm{~s}$, a steady state error of less than $5 \%$, and a maximum overshot of $20 \%$ for the step response.

Different frequency and time-domain methodscan be used in Controller synthesis. The root locus technique is applied in this paper, the discussion of which is presented below. This technique provides graphical information in the complex plane on the trajectory of the roots of the characteristic equation for variations in one or more system parameters. Since the roots placement in the complex plane control the type of the response expected to occur, the ability to present the movement of the roots in the complex plane, with the variation of one or more system parameters, turns out to be very useful.

The root locus and the closed-loop step response plot of the transfer function is defined in Fig.4.

\section{Numerical Values}

The following numerical data for a class of guided missile have been considered for MATLAB simulation [6].

\subsection{Transfer Function of Two-Loop Auto-pilot In yaw Plane}

Dynamical stability analysis is presented in the following section using Matlab software to examine the roots of the characteristic equation. Transfer function is written inmatlab in the following manner: $\mathrm{GX} 1=\mathrm{TF}$ (system).

The open loop transfer function of the autopilot for loop opened at X1 is given by,

$$
\begin{gathered}
G X 1=\frac{K_{P} G_{1}(S) G_{2}(S) G_{3}(S)}{1+G_{1}(S) G_{2}(S)}(2) \\
=\frac{K_{P} K_{q} K_{b} \omega_{a}^{2} \omega_{b}^{2}\left(1=\sigma^{2} s^{2}\right)}{\left(s^{2}+\omega_{b}^{2}\right)\left(s^{2}+2 \zeta_{a} \omega_{a} S+\omega_{a}^{2}\right)+K_{q} K_{b} \omega_{a}^{2} \omega_{b}^{2}\left(1+S T_{a}\right)}
\end{gathered}
$$

$$
\begin{aligned}
& G X 1 \\
& =\frac{-5137.832 S^{2}-17716662}{S^{4}+2542 S^{3}+3.254 S^{2}+91114264 S-253095174}
\end{aligned}
$$

The resulting of closed-loop step response is shown in Figure 3

Figure 4: Root locus and the closed-loop step response plot of the transfer function

\subsection{Lead Compensation}

Generally,the purpose of the compensator design is to satisfy both transient and steady-state specifications. In the presented root locus design, these two tasks are approached separately. First, the transient performance specifications are satisfied, using one or more stages of lead (usually) or lag compensation [7]. The transfer function of a typical lead compensator is as the following, where the zero is smaller than the pole, which means, it is closer to the imaginary axis in the complex plane.

$$
C(S)=K \frac{S-Z}{S-P}
$$

Then by clicking the Show Analysis Plot button a window entitled LTI Viewer for SISO Design Task displaying the system's closed-loop step response will open. Some characteristics of the step response can also be identified. 


\section{International Journal of Science and Research (IJSR) \\ ISSN (Online): 2319-7064}

Index Copernicus Value (2013): 6.14 | Impact Factor (2015): 6.391

Specifically, a right-click on the figure and under Characteristics to choose Settling Time. Then repeat for Rise Time[3].

\section{Simulation Results}

The simulations are carried out in MATLAB environment and the results obtained areshown in Fig.5, Fig.6 andFig.7

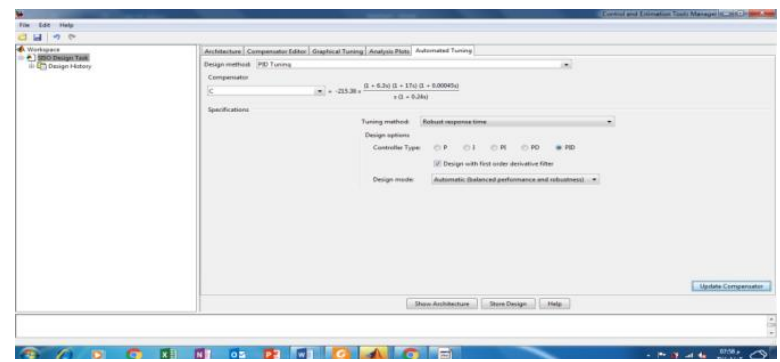

Figure 5: Control and estimation tool manager of the transfer function

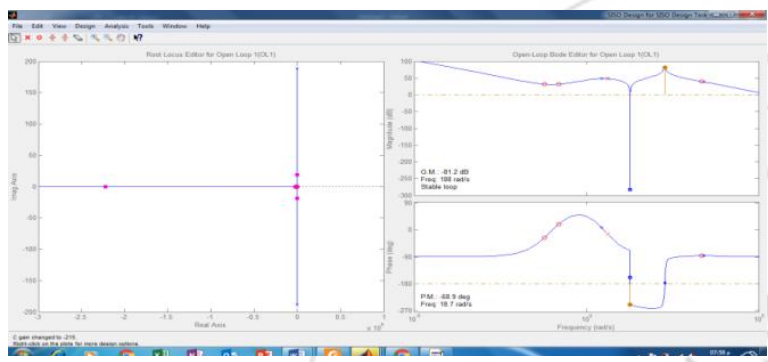

Figure 6: SISO design for the transfer function

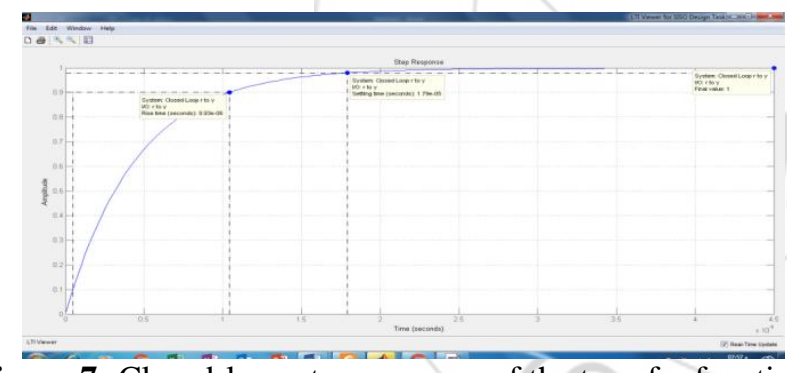

Figure 7: Closed-loop step response of the transfer function

\section{Conclusions}

The dynamical behavior of the missile two-loop autopilotis accomplished. It's shown that with selected compensator the parameters of the transfer function completely satisfy the design requirements. It can be statedthat it was completely eliminated and the overshoot value of 9.08, while settling time value around $1.79 \mathrm{e}-05 \mathrm{~s}$ and rise time value of $9.93 \mathrm{e}-06$ $\mathrm{s}$ for transfer function.

\section{References}

[1] Rami Ali Abdalla, Muawia Mohamed Ahmed, Eltahir Mohamed Hussein," Design of Missile Two-Loop Auto-Pilot Pitch Using Root Locus" International Journal Of Advances in Engineering and Management (IJAEM), Volume 1, Issue 5, November - 2014.

[2] A. Chowdhury, S. Das "Analysis and Design of Missile Two Loop Autopilot", Advance in Electronic and Electric Engineering. ISSN 2231-1297, Volume 3, Number 8 (2013), pp. 959-964.
[3] Ming Xin, S.N. Balakrishnan, " Nonlinear Missile Autopilot Design with $\theta-\mathrm{D}$ Technique" University of Missouri-Rolla, Rolla, MO 65409, Boeing Company, St. Louis, MO 63166, Naval Surface Weapon Center, Dahlgren, VA 22448-5000.

[4] Farhan A. Faruqi, ThanhLan Vu," Mathematical Models for a Missile Autopilot Design ", Weapons Systems Division Systems, Sciences Laboratory DSTO-TN-0449, August 2002.

[5] Chang-Hun Lee, Jin-Ik Lee, Byung-Eul Jun, MinGukSeo, Min-JeaTahk, Korea, " Missile Acceleration Controller Design using PI and TimeDelay Adaptive Feedback Linearization Methodology", Advanced Institute of Science and Technology (KAIST), Daejeon, 305-701, Korea.

[6] Jyoti Prasad Singha Thakur, AmitMukhopadhyay," A Simple Design Approach in Yaw Plane for Two Loop Lateral Autopilots", International Journal of Innovative Research in Science, Engineering and Technology, Vol. 2, Issue 1, January 2013.

[7] Prof. Guy Beale, " Compensator Design to Improve Steady-State Error Using RootLocus," Electrical and Computer Engineering Department, George Mason University, Fairfax, Virginia.

\section{Author Profile}

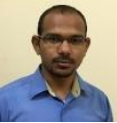

Hamimi Osama Hamimi holds a B.Sc. in control system from Al Neelain University faculty of engineering in Sudan. And post Graduates student MSC in Control System Engineering at Al Neelain University, College of Graduates Studies, in Sudan. 2007, work as lecturer in Al Neelain University for ten years.During this period, he had many administrative position in faculty of engineering. Last position was the Dean of engineer in faculty.

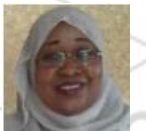

Dalia Mahmoud Assistant Professor and currentlyHead of Control Systems Department, at Al Neelain University, Faculty of engineering in Sudan. Dalia holds a Ph.D. degree and she hasmore than 10 years' Experience in higher Education filed. 